\title{
A review of non-collagenous proteins; their role in bone
}

\author{
Aiman I. Al-Qtaitat ${ }^{1}$, Saed M. Aldalaen ${ }^{2}$ \\ ${ }^{1}$ Departments of Anatomy \& Histology, Faculty of Medicine, Mutah University, Karak, Jordan \\ ${ }^{2}$ Departments of Pharmacology, Faculty of Medicine, Mutah University, Karak, Jordan \\ Email address: \\ aimanaq2000@yahoo.com (A. I. Al-Qtaitat), aldalaensm@yahoo.com (S. M. Aldalaen)
}

\section{To cite this article:}

Aiman I. Al-Qtaitat, Saed M. Aldalaen. A Review of Non-Collagenous Proteins; their Role in Bone. American Journal of Life Sciences. Vol. 2, No. 6, 2014, pp. 351-355. doi: 10.11648/j.ajls.20140206.14

\begin{abstract}
One of the unique aspects of the bone formation process is the biological mineralization of the proteinaceous matrix. The physicochemical process of mineralization is closely regulated; the precise nature of this regulation is not completely understood. The mineralization process is influenced by matrix proteins, some of which act as inhibitors and some as enhancers. Such factors include collagen mainly types I, III and VI, extracellular matrix proteins and cell attachment proteins, in addition to systemic hormones.
\end{abstract}

Keywords: Non-Collagenous Proteins, Extracellular Matrix Proteins, Biomineralization, Cell Attachment Proteins, Growth Factors

\section{Introduction}

The skeleton in higher vertebrates has two functions, one as a reservoir of inorganic elements responding to extracellular fluid homeostasis, the other as a protection of soft tissues or as a structural support permitting movement and locomotion in the gravitational environment of the Earth's surface. The structural material is a network of protein fibres bundles arranged in layers, and permeated with dense accumulations of mineral. The protein fibres provide strength and flexibility, and the mineral makes it stiff and hard. The cells of the skeleton that maintain the structure and also enable adaptation to mechanical usage are the same cells that maintain the plasma calcium homeostasis. This dual purpose suggests that effective mechanisms are essential to organize and control the number, location and efficiency of these cells in order to fulfill these disparate and sometimes conflicting roles. Activities are coordinated over wide areas of bone surface, and prolonged states of imbalance may develop.

Bone, dentin, and cementum are produced by osteoblasts, odontoblasts, and cementoblasts, respectively. Each of these cells produces a distinct set of extracellular matrix macromolecules but produces similar structural matrices predominately constructed from type I collagen fibrils [1]. The activity of osteoclasts (bone breaking cells) and osteoblasts (bone forming cells) is under the control of the systemic hormones parathyroid, calcitonin and vitamin D and cytokines generated in the bone cell microenvironment. The mineralization process is influenced by matrix proteins, some of which act as inhibitors and some as enhancers. Such factors include collagen mainly types I, III and VI and noncollagenous proteins, such as phosphoproteins, pyrophosphate, bone Gla protein, osteocalcin, proteolipids and alkaline phosphatase [2].

\section{Non-Collagenous Proteins}

In recent years, a series of noncollagenous proteins have been isolated from bone, and while their function is not always understood at least a proportion seem to be biologically active.

2.1 Phosphoproteins are proteins which are posttranslationally modified by the attachment of either a single phosphate group, or of a complex molecule. They may bind calcium and thereby act as mineral nucleators [3]. They include bone sialoprotein and proteoglycans as minor constituents of the bone matrix. Proteoglycans inhibit calcification by masking the collagen fibrils or occupying critical spaces within the fibril and thereby diminishing diffusion, chemical interaction and sequestration of calcium ions or calcium phosphate complexes [2].

2.2 Osteocalcin also known as bone $\delta$-carboxyglutamic acid-containing protein (BGLAP) is a noncollagenous protein found in bone and dentin. Osteocalcin is the most abundant noncollagenous protein in bone $[1,4]$ comprising about $20 \%$ 
of the noncollagen matrix proteins and produced by osteoblasts. It contains three $\gamma$-carboxylglutamic acid (Gla) residues that bind calcium, and it is vitamin $\mathrm{K}$-dependent. However, its physiological role in mineralization remains uncertain. It has been postulated that rather than facilitating calcification it could retard it [5], and also that it is a chemoattractant for osteoclasts. Like alkaline phosphatase osteocalcin is used clinically as a marker of osteoblast activity and serum osteocalcin is measured by radioimmunoassay [5] as a bone turnover marker.

2.3 Matrix Gla Protein (MGP), like osteocalcin, is a member of the vitamin K-dependant $\gamma$-carboxylglutamic acid (Gla) proteins. MGP is a possible regulator of extracellular matrix calcification because MGP-deficient mice exhibit spontaneous calcification of various cartilages and also of their arteries [4].

2.4 Lipid and proteolipids are acidic phospholipids that form complexes with calcium phosphate and may be involved in the mineralization process $[1,6]$.

2.5 Alkaline phosphatase is an ecto-enzyme produced by osteoblasts and linked by Robison (1952) [7], to the mineralization process. Alkaline phosphatase may be involved in the degradation phosphate esters to provide a local concentration of phosphate or it may remove pyrophosphate to enable mineralization to proceed [1, 3]. Its distribution is before rather than at the calcification front [8] supporting an indirect preparative function.

\section{Cell Attachment Proteins}

In addition to the above matrix proteins are a number of different cell attachment proteins that have the common RGD amino acid sequence (arginin-glycin-aspartic acid), which is responsible for mediating attachment of these proteins to integrins (integral membrane proteins) on the cell surface [9]. These cell attachment proteins include fibronectin, osteopontin, osteonectin, thrombospondin and several other bone sialoproteins including one called bone sialoprotein and a second called BAG (bone acidic glycoprotein) [10].

3.1 Osteopontin is relatively abundant noncollagenous sialoprotein produced by osteoblasts that has a role in osteoclast attachment and resorption $[10,11]$ and that also has calcium binding sites. Osteopontin expression is regulated by vitamin $\mathrm{D}$, which increases its secretion. It binds to integrin receptors on the osteoclast by its RGD sequence, activating the phospholipase $\mathrm{C}$ pathway in the osteoclast and increasing intracellular calcium $[1,12]$.

3.2 Osteonectin is an acidic glycoprotein involved in cell attachment. It supports bone remodelling and maintenance of bone mass in vertebrates, as shown by the poor bone status osteonectin-deficient mice [13]. Although, it is synthesized by osteoblasts, it is also synthesized by skin by fibroblasts, tendon cells and odontoblasts [3]. It binds to type I collagen and also to hydroxyapatite, and is reported to promote crystal growth in vitro. Osteonectin also increases the production and activity of matrix metalloproteinases, a function important to invading cancer cells within bone [1].
3.3 Fibronectin is an ubiquitous cell attachment protein [2, 14] made locally by bone cells but also synthesized elsewhere and brought in by the vasculature. It is uncertain if the fibronectin has a special function in bone other than to coordinate the migration, interaction and differentiation of osteoblast precursors in vitro and in vivo (Mosher, 1989 cited by Carter et al., 1991) [15].

3.4 Thrombospondin also contains calcium binding sites in addition to the RGD sequence. It also mediates cell attachment, but any particular function in bone remains unknown [2].

3.5 Bone sialoprotein (BSP), like osteopontin, is another sialoprotein that is present unique to in the skeleton unlike other similar proteins which are expressed in many tissues. It may be another nucleator of hydroxyapatite as well as providing cell attachment and unlike osteopontin the effect of vitamin D is uncertain [4]. With cell attachment may become cell activation as is the case with osteopontin and osteoclasts $[16,17]$. BSP is a significant component of the bone extracellular matrix and has been suggested to constitute approximately $8 \%$ of all non-collagenous proteins found in bone and cementum [10].

\section{Regulatory Growth Factors in Bone}

A wide range of other biologically active proteins in bone have the potential to regulate cell activity. Most of these are growth regulatory factors, including transforming growth factor $\beta \mathrm{I}$ (TGF $\beta \mathrm{I})$, and TGF $\beta \mathrm{II}$, the bone morphogenic proteins, platelet derived growth factor, colony stimulating factors, tumor necrosis factor and lymphotoxin, insulin like growth factor I (IGFI) and IGFII $[6,18]$.

4.1 Transforming growth factor $\beta$ (TGF $\beta)$, TGF $\beta \mathrm{I}$ and TGF $\beta$ II are the most abundant of all the known growth regulatory factors in bone. Moreover, bone is the major storage site of growth factors [3]. TGF $\beta$ I and TGF $\beta$ II have an identical effect on bone cells. They are powerful stimulators of bone formation, when injected subcutaneously adjacent to a bone surface $[19,20]$. However, continuous exposure impairs differentiation and the factor is required for only a transient period. TGF $\beta$ may have a chemotactic function, increasing osteoblast migration unidirectionally to sites of resorption [21], where any effect on osteoclasts still has to be clarified [1].

4.2 Fibroblast growth factors (FGFs) are a family of growth factors, with members involved in angiogenesis, wound healing, embryonic development and various endocrine signaling pathways. FGFs are mitogenic for osteoblasts [22] and may mediate the expression of TGF $\beta$ on osteoblasts. There are acidic and basic FGFs [6] and in addition, they are angiogenic with a role in neovascularization and wound healing [2].

4.3 Bone morphogenetic proteins (BMPs) or osteogenic proteins are a group of growth factors also known as cytokines and as metabologens [23]. BMPs are members of the TGF $\beta$ family that share the same capacity of simulating bone formation and at least six distinct BMPs have been 
identified. Unlike TGF $\beta$ they enhance differentiation of the osteoblast lineage in culture [6]. BMPs are used clinically to promote skeletal repair by adding them to implant material and osteogenin (BMP3) has been shown to inhibit osteoclastic resorption.

4.4 Insulin-like growth factors (IGFs), IGF-I and IGF-II, are proteins with high sequence similarity to insulin. IGFs are present in the circulation and synthesized by many tissues, including bone, where they act similarly as local regulators of cell metabolism. IGFs have long been associated with bone growth, and IGF-I stimulates mitogenesis as well as collagen synthesis in bone organ culture [24] while it also enhances bone formation in vivo. In bone IGF-I is more potent than IGF-II and its infusion causes a generalized anabolic effect and an increase in bone remodelling (Ebeling et al., 1993 cited by Favus et al., 1999) [6]. However, their clinical application to bone growth is limited as when administered systemically they cause hypoglycemia [3].

4.5 Platelet-derived growth factor (PDGF) is one of the numerous growth factors, or proteins that regulate cell growth and division. PDGF was initially isolated from blood platelets and acts in the early phase of wound repair [6]. Normal and neoplastic tissues also synthesize PDGF, and it may act as a systemic or local regulator of tissue growth. PDGF is mitogenic, it stimulates osteoblast proliferation and collagen synthesis [24] and it may also regulate osteoclastic resorption $[1,3]$.

4.6 Colony stimulating factor (CSF) also called monocytemacrophage colony stimulating factor (M-CSF), is specific for the monocyte-macrophage lineage, and is required for normal osteoclast formation in rodents during the neonatal period [6]. In the one variant (op/op) of osteopetrosis, there is impaired production of CSF and the consequence is osteopetrosis due to decreased osteoclast number; cure is by CSF treatment (Felix et al, 1990 cited by Mundy, 1995) [3].

4.7 Lymphotoxin and tumor necrosis factor (TNF) share similar receptor mechanisms and are related functionally to interleukin-1. They seem to have identical biological effects on bone cells and both stimulate osteoclastic resorption and cause hypocalcaemia when administered in vivo [25]. Lymphotoxin is released by activated T-lymphocytes and TNF by activated macrophages [6]. TNF and lymphotoxin stimulate osteoclast proliferation from precursors [25] and contribute to the bone destruction and hypocalcaemia of malignancy. TNF is rarely produced by solid tumors; however it may be produced by normal host cells stimulated by the presence of a tumor. It has been associated with chronic inflammatory diseases, where accumulation of inflammatory cells occurs adjacent to bone surfaces [2, 3] as occurs in patient with periodontal disease, rheumatoid arthritis and cholesteatoma of the ear. TNF may also be involved in postmenopausal bone loss.

4.8 Prostaglandins (enzymatically oxygenated derivatives of free arachidonic acid) are a group of hormone-like lipid compounds that are derived enzymatically from fatty acids and have important functions in the animal body. Prostaglandins have multiple effects on bone cells, and sometimes opposite effects in different species, making their role in bone physiology difficult to discern. They are powerful bone resorbing factors in organ culture and are produced by monocytes found adjacent to endosteal bone surfaces, perhaps regulating osteoclast activity [26]. They are apparently locally active as stimulators of both bone formation and resorption but may conversely have inhibitory effects on fully differentiated osteoblasts and osteoclasts [2, 27]. The most potent are prostaglandins of the E-series and the 13, $14 \mathrm{H} 2$ compounds [3]. They may also mediate other factors in bone e.g. 1, 25 dihydroxyvitamin $\mathrm{D}_{3}$ and plateletderived growth factor which generate endogenous prostaglandin synthesis causing resorption in cultured mouse calvaria [27]. This bone resorption is inhibited by indomethacin, a drug that inhibits prostaglandin synthesis.

\section{Hormonal Control}

The systemic hormones effecting bone mineral content are parathyroid hormone, 1,25 dihydroxyvitamin D3 and calcitonin, all of which influence osteoclast activity. They are under negative feed back control and are regulated by extracellular fluid calcium concentration. Also influencing bone cell function but not under the negative feedback control of extracellular fluid calcium are estrogen and androgen, glucocorticoids, thyroid hormone and growth hormone where cytokines often mediate including interleukin-1, interleukin-1 receptor agonist, interleukin-4, interleukin-6 and interferon- $\gamma$.

5.1 Parathyroid hormone (PTH) is a polypeptide produced by the parathyroid glands that maintains calcium homeostasis, because of its stimulatory effects on osteoclastic resorption and on the renal reabsorption of calcium. It exhibits both anabolic and catabolic effects, depending in whether it is administered intermittently or continuously respectively [4]. This enabled its exploitation as a treatment for osteoporosis by giving low doses intermittently when the primary effect is increased bone formation more commonly, PTH stimulating is known for the differentiation of progenitors into mature multinucleated osteoclasts [6] and increased remodeling which is a postmenopausal characteristic.

5.2 Calcitonin is a polypeptide hormone secreted by the parafollicular cells of the thyroid gland. It causes a shortfall in plasma calcium, by inhibiting osteoclastic bone resorption [28] causing a transient contraction of the osteoclast cell membrane [29]; it also promotes calcium excretion and this effect on homeostasis usually last 24-48 hours [30]. For this reason it has been suggested that calcitonin has no chronic effect on calcium homeostasis, but rather serves as a shortterm regulator to maintain normal calcium following large calcium-containing meals when it is also influenced by gastrointestinal hormones such as gastrin.

5.3 Vitamin $D$ and 1, 25 dihydroxyvitamin D3, are the major regulators of calcium homeostasis and vitamin $D$ protects the organism from calcium deficiency via its effect on the intestine, kidney, parathyroid gland and bone. The most active vitamin D molecule is 1, 25 dihydroxyvitamin 
D3 which is formed after two consecutive hydroxylations of the skin-derived vitamin D (cholecalciferol), in the liver and kidney. Vitamin D deficiency due to dietary lack, sunshine deficiency or malabsorption of fat-soluble vitamins causes impaired mineralization of osteoid tissue and growth plates producing rickets in children and osteomalacia in adults. In contrast, excess vitamin D ingestion leads to an increased bone resorption and hypocalcaemia [4]. The effect of 1, 25 dihydroxyvitamin D3 on osteoclastic bone resorption is apparently synergistic with PTH [31]. 1, 25 dihydroxyvitamin D3 also affects osteoblast function and in culture it inhibits bone collagen synthesis in the short term [32]. It has other significant effects on calcium homeostasis, since it increases the absorption of calcium from the gut and like PTH stimulates renal tubular calcium reabsorption.

\section{Other Cytokines Related to Bone}

6.1 Interleukin-1 (IL-1) is a cytokines produced by osteoclasts and a variety of related cell types including, monocytes, macrophages and marrow stroma cells [4]. IL-1 stimulates osteoclast precursor and can induce hypocalcaemia. It also has growth-stimulatory effects on osteoblastic cells, but inhibits their differentiated function [1, 33]. There are two IL-1 molecules IL- $1 \alpha$ and IL-1 $\beta$ their similar effects on bone are mediated through the same receptor [6], Interleukin-1 receptor agonist (IL-1RA) by blocking the IL-1 receptor molecules [34].

6.2 Interleukin-4 (IL-4) is a multifunctional lymphokine produced by activated T-lymphocytes, which inhibits bone resorption in organ culture and the formation of osteoclasts from precursors [3]. However, transgenic mice overexpressing IL-4 paradoxically develop an osteopenic syndrome similar to osteoporosis [35], the high levels apparently inhibiting bone formation.

6.3 Interleukin-6 (IL-6) is also a multifunctional cytokine and, unlike other cytokines, its production in bone is enhanced by osteotropic hormones such as PTH and 1,25 dihydroxyvitamin D3 [36], causing hypocalcaemia. It increases osteoclasts from precursors and doubles the number of multinucleated cells in human marrow cultures [37].

6.4 Interferon- $\gamma$ is a powerful inhibitor of osteoclasts, inhibiting their formation and differentiation from precursors, while having no morphological effect on mature preformed osteoclasts [6].

In summary, one of the unique aspects of the bone formation process is the biological mineralization of the proteinaceous matrix. Following the production of osteoid tissue (non-mineralized matrix protein) by the osteoblasts, it is mineralized in an orderly and controlled manner at the calcification front. Although it is evident that the physicochemical process of mineralization is closely regulated, the precise nature of this regulation is not completely understood. The activity of osteoclasts and osteoblasts is under the control of the systemic hormones parathyroid, calcitonin and vitamin $\mathrm{D}$ and cytokines generated in the bone cell microenvironment. The mineralization process is influenced by matrix proteins, some of which act as inhibitors and some as enhancers.

\section{References}

[1] George A and Veis A. Phosphorylated proteins and control over apatite nucleation, crystal growth, and inhibition. Chem. Rev. 2008; 108: 4670-4693.

[2] Nudelman F, Lausch AJ, Sommerdijk NAJM and Sone ED. In vitro models of collagen biomineralization. Journal of Structural Biology 2013: 183: 258-269.

[3] Mundy GR. Bone Remodeling and its Disorders. Martin Dunitz, London, 1995.

[4] Massaro EJ and Rogers JM. The Skeleton: Biochemical, Genetic, and Molecular Interactions in Development and Homeostasis. Humana Press, Totowa, New Jersey, 2004.

[5] Price PA, Williamson MK and Haba T. Excessive mineralization with growth plate closure in rats on chronic warfarin treatment. Proceeding National Academy of Science 1982; 79: 7734-7738.

[6] Favus JM. Primer on the Metabolic Bone Diseases and Disorders of Mineral Metabolism. Lippincott Williams and Wilkins. Philadelphia, USA, 1999.

[7] Robinson RA. An electron microscopic study of the crystalline inorganic component of bone and its relationship to the organic matrix. Journal of Bone and Joint Surgery 1952; 34A: $389-434$.

[8] Aaron JE. Alkaline phosphatase, vesicles and calcification. Metabolic Bone Disease Related Research 1980; 2S: 117-125.

[9] Baron R, Chakraborty M and chatterjee D. The biology of the osteoclast. In: Physiology and Pharmacology of Bone. Handbook of Experimental Pharmacology. Editors Mundy GR and Martin TJ. Springer-Verlag, Berlin 1993; 111-147.

[10] Vincent K and Durrant MC. "A structural and functional model for human bone sialoprotein". Journal of Molecular Graphics and Modelling 2013; 39: 108-117.

[11] Reinholt FB, Hultenby K, Oldberg A and Heinegard D. Osteopontin- a possible anchor of osteoclasts to bone. Proceeding National Academy of Science 1990; 87: 44734475 .

[12] Tanaka H, Hruska KA and Seino. Disassociation of the macrophage-maturational effect of vitamin D from respiratory burst priming. Journal of Biology and Chemistry 1991; 266: 888-892.

[13] Delany AM, Amling M, Priemel M, Howe C, Baron R and Canalis E. Osteopenia and decreased bone formation in osteonectin-deficient mice. Journal of Clinical Investigation 2000; 105: 915-923.

[14] Weiss RE and Reddi AH. Synthesis and localization of fibronectin during collagenous matrix-mesenchymal cell interaction and differentiation of cartilage and bone in vivo. Proceeding National Academy of Science 1980; 77: 2074.

[15] Carter DH, Sloan P and Aaron JE. Immunlocalization of collagen type I and III, tenascin and fibronectin in intramembranous bone. The Journal of Histochemistry and Cytochemistry 1991; 39: 599-606. 
[16] Herring GM. The organic matrix in bone. In The Biochemistry and Physiology of Bone, Academic Press: New York 1972; 127.

[17] Fisher LW, Whitson SW and Avioli LV. Matrix sialoprotein of developing bone. Journal of Biology and Chemistry 1983; 258: 12723.

[18] Hauschka PV, Mavrakos AE and Iafrati MD. Groth factors in bone matrix, isolation of multiple types by affinity chromatography on heparin-sepharose. Journal of Biology and Chemistry 1986; 112: 83-88.

[19] Noda $M$ and Camillier JJ. In vivo stimulation of bone formation by transforming growth factor $\beta$. Endocrinology $1989 ; 124: 2991-2994$.

[20] Mackie EJ and Trechsel U. Stimulation of bone formation in vivo by transforming growth factor $\beta$-remodeling of woven bone and lack of inhibition by indomethacin. Bone 1990; 11:295-300

[21] Pfeilschifter J, Wolf O and Naumann A. Chemotactic response of osteoblast-like cells to transforming growth factor $\beta$. Journal of Bone and Mineral Research 1990; 5:825-830.

[22] Rodan SB, Wesolowski G and Thomas K. Growth stimulation of rat calvaria osteoblastic cells by acidic fibroblast growth factor. Endocrinology 1987; 121: 1917-1923.

[23] Reddi, AH and Reddi A. "Bone morphogenetic proteins (BMPs): from morphogens to metabologens". Cytokine \& growth factor reviews 2009; 20 (5-6): 341-2.

[24] Canalis EM. Effect of insulin-like growth factor I on DNA and protein synthesis in cultured rat calvaria. Journal of Clinical Investigations 1980; 66: 709-719

[25] Garrett IR, Durie BGM and Nedwin GE. Production of the bone resorping cytokine lymphotoxin by culture human myeloma cells. New England Journal of Medicine 1987; 317: 526-532.

[26] Dominguez JH and Mundy GR. Monocytes mediate osteoclastic bone resorption by prostaglandin production. Calcified Tissue International 1980; 31: 29-34.
[27] Raisz LG. Prostaglandins and bone: physiology and pathophysiology. Osteoarthritis 1999; 7: 419-421.

[28] Friedman J, Au WYW and Raisz LG. Response of fetal rat bone to thyrocalcitonin in tissue culture. Endocrinology 1986; 82: $149-156$.

[29] Chambers TJ and Ali HN. Inhibition of osteoclastic motility by prostaglandins. Journal of Pathology 1983; 139: 383-393.

[30] $\mathrm{Au}$ WYW. Calcitonin treatment of hypercalcemia due to parathyroid carcinoma: synergistic effect of prednisolone on long term treatment of hypercalcemia. Archive of International Medicine 1975; 135: 1594-1597.

[31] Garabedian M, Tanaka Y and Holick MF. Response of intestinal calcium transport and bone calcium mobilization to 1,25 dihydroxyvitamin $\mathrm{D}_{3}$ in thyroparathyroidectemised rat. Endocrinology 1974; 94: 1022-1027.

[32] Raisz LG, Kream BE and smith MD. Comparison of the effect of vitamin D metabolites on collagen synthesis and resorption of fetal rat in organ culture. Calcified Tissue International 1980; 32: 135-138.

[33] Smith D, Gowen M and Mundy GR. Effects of interferon gamma and other cytokines on collagen synthesis in fetal rat bone cultures. Endocrinology 1987; 120:2494-2498.

[34] Arend WP, Joslin FG and Thombson RC. An IL-1 inhibitor from human monocytes. Production and characterization of biologic properties. Journal of Immunology 1989; 15: 18511858.

[35] Lewis BD, Liggit D, and TEitelbaum S. Mechanism of osteoporosis in IL-4 transgenic mice. Journal of Bone and Mineral Research 1992; 7 S no 20.

[36] Feyen JHM, Elford P and Dipadova FE. Interleukin-6 is produced by bone and modulated by parathyroid hormone. Journal of Bone and Mineral Research 1989; 4:633-638.

[37] Kurihara N, Bertolini D and Suda T. Interleukin-6 stimulates osteoclast-like multinucleated cell formation in long term human marrow cultures by inducing IL-1 release. Journal of Immunology 1990; 144: 426-430. 